Als Klinikchef hat sich Müller besonders für eine bessere Betreuung der betagten psychiatrischen Patienten eingesetzt und für diese Stiefkinder der Psychiatrie - «les parents pauvres de la médecine mentale»-eine besondere Abteilung eingerichtet.

Christian Müller hat seit jeher ein lebendiges Interesse für die Geschichte seines Faches gezeigt. Er hat junge Ärzte zu historischen Studien angeregt und betätigt sich weiterhin selbst auf diesem Gebiet. Zusammen mit seiner Frau teilt er seine Zeit zwischen Bern, wo er noch praktiziert, und Onnens am waadtländischen Ufer des Neuenburgersees. In dieser weiten, großzügigen Landschaft soll er, wie man hört, eine Geschichte der Psychiatrie ausgearbeitet und schon nahezu vollendet haben, «un ouvrage plein d'anecdotes», also den Leser ansprechend - aber gewiß nicht nur das!

Der Vorstand der SGGMN - den er mit ruhiger Überlegenheit präsidiert und die Redaktion des Gesnerus gratulieren Christian Müller herzlich. Er ist eine Verkörperung vorbildlicher, wohltuender Lebensweisheit - möge er sich und andere noch lange damit erfreuen!

Urs Boschung

H.M. Koelbing

\title{
Un prix important
}

La Société de Physique et d'Histoire naturelle de Genève (SPHN) a l'honneur de vous informer qu'elle attribue un prix intitulé:

\section{Prix Marc-Auguste Pictet}

Ce Prix, destiné en principe à un jeune chercheur, récompensera un travail marquant, inédit ou récemment publié, dans le domaine de l'Histoire des sciences.

Son montant est de Sfr.12000.- et peut être partagé.

Sont habilités à le recevoir, des candidats suisses ou étrangers, de niveau universitaire. Les candidatures doivent être envoyées jusqu'au $1^{\text {er }}$ février 1992 à l'adresse suivante:

Président de la SPHN

Muséum d'Histoire naturelle

Case postale 434

CH-1211 Genève 6

Suisse 
Les textes complets des travaux, accompagnés d'un résumé et d'un curriculum vitae, doivent lui être soumis dans ce délai, en deux exemplaires. Un exemplaire des travaux primés restera propriété de la SPHN.

Ces textes peuvent être rédigés en français, allemand, italien ou anglais. Dans ces trois derniers cas le résumé sera traduit en français et comprendra une douzaine de pages, soit 4000 mots ou 20000 signes environ.

Le thème choisi pour le Prix en 1992 concerne l'Histoire de la chimie.

Une commission est chargée d'examiner les candidatures.

Le Président de la SPHN

Marc-Auguste Pictet (1752-1825) fut l'un des huit fondateurs de la Société de Physique et d'Histoire naturelle de Genève, en 1790. Il fut aussi, en 1796, l'un des créateurs de la revue littéraire et scientifique internationale «La Bibliothèque Britannique», ancêtre de l'actuelle revue «Archives des Sciences» éditée par la SPHN.

Il succéda à H. B. de Saussure dans la chaire de philosophie (naturelle) de l'Académie de Genève, puis à Jacques André Mallet dans celle d'astronomie assumant la direction de l'Observatoire de Genève pendant 30 ans. Membre de la Société Royale de Londres et de l'Académie des Sciences de Paris, il fut l'un des savants les plus en vue de son époque.

\section{Eine Zuschrift}

Entgegnung auf die Rezension von Cornelia Isler-Kerényi über das Buch von Hildegard Sobel, Hygieia. Die Göttin der Gesundheit, WB Darmstadt 1990 (Gesnerus 47, 1990, 394.f.)

Was der Verfasserin der Rezension offenbar vorschwebt, «eine wirkliche verehrungswürdige religiöse Gestalt zu entdecken», war nicht die Absicht meiner Abhandlung. Mein Anliegen war es, die archäologisch relevanten Objekte zu erfassen und daraus die Typologie der Hygieia und deren Wandlung im Verlaufe von acht Jahrhunderten zu beschreiben. Die Schriftquellen wurden berücksichtigt.

Wenn es auch einige literarische Belege für einen älteren selbständigen Kult der Hygieia gibt und auch eine Athena Hygieia des 5. Jh. vor Chr. bezeugt ist, tritt die Hygieia, die Kultgefährtin des Asklepios, erst gegen Ende des 5. Jh. vor Chr. in Erscheinung und entleiht ihre Ikonographie von der anderer Göttinnen. Auch der Paean des Ariphron stammt erst aus dem 4. Jh. vor Chr. Aus dem Aussehen, den Attributen und Beziehungen Hygieias neue Einsichten in ihr Wesen zu gewinnen, halte ich für gewagt, wenn man 